\title{
Risk of Monozygotic Twins After Assisted Reproduction: A Population-Based Approach
}

\author{
Fabio Parazzini, ${ }^{1,2}$ Sonia Cipriani, ${ }^{1,2}$ Stefano Bianchi, ${ }^{1,3}$ Camilla Bulfoni, ${ }^{4}$ Renata Bortolus, ${ }^{5}$ and \\ Edgardo Somigliana ${ }^{2}$ \\ ${ }^{1}$ Department of Clinical Sciences and Community, University of Milan, Milan, Italy \\ ${ }^{2}$ Department Mother and Child, Maggiore Hospital of Milan, University of Milan, Milan, Italy \\ ${ }^{3}$ IRCCS Multimedica Ospedale S Giuseppe, Divisione di Ostetricia e Ginecologia, Milano, Italy \\ ${ }^{4}$ AO Ospedale Maggiore Niguarda, Milano, Italy \\ ${ }^{5}$ Office for Research Promotion, Department of the Hospital Management and Pharmacy, Verona University Hospital, \\ Verona, Italy
}

\begin{abstract}
Background: Recent studies have suggested that ovarian stimulation and assisted reproductive techniques (ART) may increase the frequency of monozygotic twins. In this article, we present the analysis of the estimated frequency of twin deliveries following in vitro fertilization (IVF) in Lombardy during the period 2010-2014 for a total of 450,949 pregnancies. Method: This is a population-based study using data from the regional data base of Lombardy, a northern Italian region with a population of about 10 million inhabitants. During the considered period, a total of 461,424 single or multiple births were registered in Lombardy. After exclusion of triplets or more pregnancies, the total number of twin deliveries, in separate strata of like and unlike sex pregnancies twin deliveries, were obtained and the rate of twin deliveries was computed according to spontaneous and non-spontaneous conception and type of ART. Further, estimates of dizygotic or monozygotic twin births were calculated using Weinberg's methods. Results: The frequency of twins deliveries was 1.24/100 deliveries after natural conception and 20.05 after assisted conception. The estimated rates of monozygotic twins was 0.45 and $0.72 / 100$ ( $95 \% \mathrm{Cl}: 0.58-0.91$ ) deliveries after natural and assisted conception, respectively. This difference was statistically significant $(p<.05)$. Conclusion: the present population based study suggests that the risk of monozygotic twin is about $60 \%$ higher among assisted than after natural conception.
\end{abstract}

Keywords: assisted reproduction, twins, monozygotic

Recent studies have suggested that ovarian stimulation and ART may increase the frequency of monozygotic twins (Delrieu et al., 2012; Franasiak et al., 2015; Gee et al., 2014; Kanter et al., 2015; Knopman et al., 2010; 2014; Luke et al., 2014; Nakasuji et al., 2014; Osianlis et al., 2014; Ren et al., 2013; Tocino et al., 2015; Vitthala et al., 2009).

Published data on this issue are, however, not robust and largely inconsistent (Sobek et al., 2015). While the rate of monozygotic twins in natural conceptions has been reported to be about 0.4\% (Hall, 2003; Imaizumi \& Nonaka, 1997), the rates reported in IVF pregnancies vary between 0.7 and 13\% (Delrieu et al., 2012; Gee et al., 2014; Knopman et al., 2010; 2014; Nakasuji et al., 2014; Osianlis et al., 2014; Ren et al., 2013; Tocino et al., 2015; Vitthala et al., 2009). Data obtained from case series of monozygotic twins are also heterogeneous. The reported proportions of IVF pregnancies among monozygotic twins ranges from $8 \%$ to $32 \%$, thus higher than the background rate of IVF conceptions in the referring populations, but still epidemiologically imprecise (Chow et al., 2001; Ghalili et al., 2013; Ortibus et al., 2009; Sperling et al., 2006).

All published data are based on limited clinical series and no population-based studies have been published on this issue. In this article, we present the analysis of frequency of twin deliveries after IVF in Lombardy during the period $2010-14$ for a total of 450,949 pregnancies. The frequency of dizygotic and monozygotic twin is estimated

RECEIVED 17 September 2015; ACCEPTED 3 November 2015. First published online 8 January 2016.

ADDRESS FOR CORRESPONDENCE: Fabio Parazzini, Dipartimento di Scienze Cliniche e di Comunità, Università degli Studi di Milano, via Commenda 12-20122 Milan, Italy. E-mail address: fabio.parazzini@unimi.it 
using Weinberg's rule, assuming that sex gender is independently distributed in dichorionic twins. The difference between the total number of twins and two times the number of discordant twins thus provides an estimation of the number of monozygotic twins.

\section{Methods}

In Lombardy, a standard form is used to register all births and neonatal discharges from public or private hospitals. All admissions and discharges are codified according to the International Classification of Diseases 9th edition Clinical Modification (ICD-9-CM), Italian version. For all deliveries, information was available for maternal age, maternal country of birth, and reason for admission. Neonatal information included live birth/stillbirth status and sex. Further, at delivery, a specific form is filled out by midwifes that includes information on the pregnancy (single or multiple), maternal characteristics, type of conception (spontaneous/non-spontaneous (i.e., after ART or medically induced ovulation only), course of pregnancy, delivery, and maternal outcome at birth (provided on the CedAP database). A more detailed description of methodology is reported elsewhere (Parazzini et al., 2015).

On the basis of these data, the total number of twin deliveries, including a separate strata of like and unlike sex pregnancies, were obtained and the rate of twin deliveries was computed according to spontaneous and non-spontaneous conception and type of ART. Further, estimates of dizygotic or monozygotic twin births were calculated using Weinberg's methods (Weinberg, 1901). Briefly, it was assumed that, in contrast to monozygotic pregnancies, sex gender is independently distributed in dichorionic twins. The difference between the total number of twins and two times the number of discordant twins thus provides an estimation of the number of monozygotic twins. At all stages of the analysis the unit of the study was the pregnancy and not the child(ren).

During the period under consideration, a total of 461,424 single or multiple births were registered in Lombardy; 2,882 births were excluded from the analysis because of a lack of information concerning the sex of newborns or type of conception (spontaneous or assisted). Of the remaining 459,142 births, 612 were triplets and 16 were quadruplets, and these births are not considered for the present analysis. The analysis was thus conducted on 458,934 births $(450,949$ deliveries).

Analysis of an administrative, anonymous database does not require ethics approval in Italy. All data were anonymous.

\section{Results}

A total of 10,554 deliveries $(2.3 \%)$ were reported after ART/medically induced ovulation only. Techniques used were medically induced ovulation only ( $n=659,6.2 \%)$, intrauterine insemination (IUI; $n=957,9.1 \%$ ), IVF $(n=3,319,31.5 \%)$, ICSI $(n=4,391,41.6 \%)$ and others - unspecified ( $n=1,228,11.6 \%)$.

Table 1 shows the distribution of single and twin births after natural and assisted conception observed in Lombardy during the study period, according to selected factors. In comparison with women who conceived naturally, mothers who had assisted conception were older, more educated and were more likely to be an Italian citizen $(p<.001)$. The father of the child was more frequently older and more educated.

Overall, the frequency of twins deliveries was 1.24/100 deliveries after natural conception and 20.05/100 after assisted conception. The estimated rates of monozygotic twins was 0.45/100 (95\% CI: $0.42-0.47)$ and 0.72/100 (95\% CI: $0.58-0.91)$ deliveries after natural and assisted conception, respectively. This difference was statistically significant $(p$ $<.05$ ). We have further estimated the monozygotic twins rates according to the type of assisted techniques (ovulation induction and IUI were grouped for sample size reasons; see Table 2). The estimated rate of monozygotic twins in the ovulation induction/ IUI, IVF, and ICSI groups were 0.77 (95\% CI: 0.62-0.93), 0.58 (95\% CI: 0.52-0.65), and 1.63 (95\% CI: 1.27-2.13) respectively. All of these results were significantly higher when compared to natural conceptions $(p<.05$ for all three comparisons). Intergroup comparisons documented that ICSI was associated with a statistically significant higher risk when compared to both ovulation induction/IUI $(p \leq .05)$ and to $\operatorname{IVF}(p \leq .05)$. Conversely, the rates observed in these latter two groups did not significantly differ.

\section{Discussion}

Assisted conceptions are associated with a higher rate of monozygotic twins. However, the magnitude of the association is smaller compared to some worrying estimations provided by some case series. The rate is higher and more clinically relevant when focusing on the ICSI group.

A potential main limitation of this analysis is the quality of information reported in routine statistics on type of conception. However, this possible concern is unlikely to affect our results. The information considered in this analysis was included by law in an administrative database that registers all hospital admissions in private and public hospitals. These data should be considered totally representative of newborns admitted to the hospitals in the region, without any bias. Further, the frequency of births after ART reported in the regional database is largely consistent with the data reported in the Italian Registry of ART (http://www.iss.it/rpma/). Moreover, the type of assisted conception is also consistent with the available data on the clinical practice in Lombardy. In any case, any misclassification should tend to reduce the estimate frequency of twin deliveries among assisted conception, and it is unlikely 
TABLE 1

Characteristics of Mother and Father According to Mode of Conception and Type of Pregnancy

\begin{tabular}{|c|c|c|c|c|c|c|c|c|c|c|}
\hline \multirow{2}{*}{ Maternal age } & \multicolumn{4}{|c|}{ Spontaneous conception } & \multicolumn{4}{|c|}{ Assisted conception } & \multirow[b]{2}{*}{$p$ value* } & \multirow[b]{2}{*}{$p$ value** } \\
\hline & \multicolumn{2}{|c|}{ Singleton $n=434,934$} & \multicolumn{2}{|c|}{ Twins $n=5,461$} & \multicolumn{2}{|c|}{ Singleton $n=8,438$} & \multicolumn{2}{|c|}{ Twins $n=2,116$} & & \\
\hline & & & & & & & & & & \\
\hline$\leq 24$ & 45,832 & 99.1 & 422 & 0.9 & 77 & 91.7 & 7 & 8.3 & & \\
\hline $25-29$ & 95,800 & 98.9 & 1081 & 1.1 & 541 & 80.0 & 135 & 20.0 & & \\
\hline $30-34$ & 147,731 & 98.7 & 1897 & 1.3 & 2,249 & 80.1 & 559 & 19.9 & & \\
\hline $35-39$ & 114,861 & 98.7 & 1555 & 1.3 & 3,348 & 80.3 & 822 & 19.7 & & \\
\hline $40+$ & 29,373 & 98.4 & 488 & 1.6 & 2203 & 78.9 & 590 & 21.1 & & \\
\hline Missing & 1,337 & 98.7 & 18 & 1.3 & 20 & 87.0 & 3 & 13.0 & & \\
\hline \multicolumn{11}{|l|}{ Mean $(S D)$} \\
\hline Maternal education & & & & & & & & & $<.0001$ & $<.0001$ \\
\hline University & 121,367 & 98.7 & 1593 & 1.3 & 3,527 & 79.7 & 896 & 20.3 & & \\
\hline High school & 188,849 & 98.7 & 2408 & 1.3 & 3,688 & 80.1 & 916 & 19.9 & & \\
\hline $\begin{array}{l}\text { Primary/intermediate } \\
\text { school or no } \\
\text { education }\end{array}$ & 123,075 & 98.8 & 1450 & 1.2 & 1,215 & 80.0 & 303 & 20.0 & & \\
\hline Missing & 1,643 & 99.4 & 10 & 0.6 & 8 & 88.9 & 1 & 11.1 & & \\
\hline Paternal age & & & & & & & & & $<.0001$ & $<.0001$ \\
\hline$<24$ & 14,023 & 99.2 & 114 & 0.8 & 24 & 88.9 & 3 & 11.1 & & \\
\hline $25-29$ & 50,886 & 99.0 & 505 & 1.0 & 204 & 85.7 & 34 & 14.3 & & \\
\hline $30-34$ & 120,702 & 98.8 & 1435 & 1.2 & 1,340 & 79.9 & 337 & 20.1 & & \\
\hline $35-39$ & 135,044 & 98.7 & 1774 & 1.3 & 3,019 & 80.7 & 721 & 19.3 & & \\
\hline $40-44$ & 69,038 & 98.7 & 930 & 1.3 & 2,238 & 79.2 & 589 & 20.8 & & \\
\hline $45+$ & 27,674 & 98.5 & 410 & 1.5 & 1,316 & 79.2 & 346 & 20.8 & & \\
\hline Mean (SD) & 17,567 & 98.4 & 293 & 1.6 & 297 & 77.5 & 86 & 22.5 & & \\
\hline Mother citizenship & & & & & & & & & $<.0001$ & $<.0001$ \\
\hline Italian & 305,946 & 98.7 & 3926 & 1.3 & 7432 & 80.0 & 1862 & 20.0 & & \\
\hline Not Italian & 128,642 & 98.8 & 1525 & 1.2 & 1,003 & 80.0 & 251 & 20.0 & & \\
\hline Missing & 346 & 97.2 & 10 & 2.8 & 3 & 50.0 & 3 & 50.0 & & \\
\hline Nulliparous & & & & & & & & & $<.0001$ & $<.0001$ \\
\hline No & 206,150 & 98.8 & 2473 & 1.2 & 1,275 & 84.4 & 235 & 15.6 & & \\
\hline Yes & 221,402 & 98.7 & 2910 & 1.3 & 6,731 & 79.1 & 1,774 & 20.9 & $<.0001$ & $<.0001$ \\
\hline Missing & 7,382 & 99.0 & 78 & 1.0 & 432 & 80.1 & 107 & 19.9 & & \\
\hline Type of ART & & & & & & & & & - & - \\
\hline Ovulation induction only & - & & - & & 594 & 90.1 & 65 & 9.9 & & \\
\hline IUI & - & & - & & 839 & 87.7 & 118 & 12.3 & & \\
\hline ICSI & - & & - & & 3,429 & 78.1 & 962 & 21.9 & & \\
\hline IVF & - & & - & & 2,588 & 78.0 & 731 & 22.0 & & \\
\hline Other not specified & - & & - & & 988 & 80.5 & 240 & 19.5 & & \\
\hline
\end{tabular}

Note: ${ }^{*} p$ value of chi-square association between mode of conception and mother and father characteristics; ${ }^{* *} p$ value of chi-square association between type of pregnancy (single/twin) and mother and father characteristics.

TABLE 2

Twin Deliveries Rates According to Type of Conception

\begin{tabular}{lllllll}
\hline & $\begin{array}{l}\text { Total } \\
\text { deliveries } \\
\text { No. }\end{array}$ & $\begin{array}{l}\text { Twin } \\
\text { deliveries } \\
\text { No. }\end{array}$ & $\begin{array}{l}\text { Unlike } \\
\text { sex twin } \\
\text { deliveries } \\
\text { No. }\end{array}$ & $\begin{array}{l}\text { Twin } \\
\text { deliveries } \\
\text { rate/100 } \\
\text { deliveries }\end{array}$ & $\begin{array}{l}\text { Monozygotic } \\
\text { rate/100 } \\
\text { deliveries }\end{array}$ & $\begin{array}{l}\text { Cl 95\% Of } \\
\text { monozygotic } \\
\text { rate/100 } \\
\text { deliveries* }\end{array}$ \\
\hline Natural conception & 440,395 & 5,461 & 1,743 & 1.24 & 0.45 & $0.42-0.47$ \\
Any type of assisted conception & 10,554 & 2,116 & 1,020 & 20.05 & 0.72 & $0.58-0.91$ \\
First level techniques ** a & 1,433 & 183 & 86 & 12.77 & 0.77 & $0.62-0.95$ \\
FIVET $^{\circ}$ & 2,588 & 731 & 358 & 22.02 & 0.58 & $0.52-0.65$ \\
ICSI $^{\circ}$ & 3,429 & 962 & 453 & 21.91 & 1.63 & $1.27-2.13$ \\
\hline Note: $^{*}$ Based on Poisson's approximation; **IUI and ovulation induction. ${ }^{2}$ The sum of first level techniques, FIVET, and ICSI does \\
not reach the total of 'Any type of assisted conception' due to missing data.
\end{tabular}

that misreporting may differ in case of delivery of like or unlike sex children. Finally, it is worthwhile noting that the calculated rate of monozygotic twin in natural pregnancies emerging from our analysis $(0.45 / 100$ deliveries) is perfectly in line with the previous population-based estimations (Gee et al., 2014; Hall, 2003; Imaizumi \& Nonaka, 1997), thus indirectly supporting the quality of our data.
The use of Weinberg's method to assess the rate of monozygotic twins may be another matter of concern. The validity of the method has been recently confirmed in the general population (Fellman \& Eriksson, 2006; Hardin et al., 2009) but confirmation in ART children is lacking. Inferences of the validity of the method in the general population to ART children should be made with caution because 
potential deviation of the sex ratio might exist (Blickstein, 2005).

Further, in this study, we observed the well-recognized association between older maternal age and frequency of ART. It is conceivable that women conceiving with ART and those conceding naturally may differ in some characteristics that can be related to the risk of monozygotic twin births. In our population, women who did not conceive spontaneously were older, but the risk of spontaneous monozygotic twins is not related to maternal age (Bortolus et al., 1999).

Another interesting finding of this study is the analysis of the frequency of monozygotic twins after different ART techniques. Albeit it is debated, there is some evidence suggesting a role for ovarian hyperstimulation, prolonged culture up to the blastocysts stage, culture medium, number of embryos transferred, and breach of the zona pellucida with ICSI or assisted hatching (Chang et al., 2009; Delrieu et al., 2012; Franasiak et al., 2015; Kanter et al., 2015; Knopman et al., 2010; 2014; Luke et al., 2014; Nakasuji et al., 2014; Ren et al., 2013; Vitthala et al., 2009). In other words, differences may be a consequence of the specific ART technique used and of local differences in clinical and laboratory protocols (Vitthala et al., 2009). Our study tends to concur with the growing literature, albeit it is not homogeneous, supporting a detrimental effect of the ICSI procedure. Unfortunately, since this is a register-based study, there is no possibility of investigating the independent role of specific laboratory procedures, such as, for example, the cultured media used, the prolonged culture, and the assisted hatching. From a clinical perspective, the observation of a possible increased risk of monozygotic twins following ICSI should be taken into consideration in the current debate regarding the worldwide and non-evidence based growth of ICSI use (Kanter et al., 2015).

In conclusion, the present population-based study suggests that the risk of monozygotic twin is about $60 \%$ higher among assisted than after natural conception and that the use of ICSI may play a critical role. Large studies based on clinical diagnosis of monozygotic may confirm this finding on a sound basis.

\section{References}

Blickstein, I. (2005). Estimation of iatrogenic monozygotic twinning rate following assisted reproduction: Pitfalls and caveats. American Journal of Obstetrics and Gynecology, 192, 365-368.

Bortolus, R., Parazzini, F., Chatenoud, L., Benzi, G., Bianchi, M. M., \& Marini, A. (1999). The epidemiology of multiple births. Human Reproduction Update, 5, 179-187.

Chang, H. J., Lee, J. R., Jee, B. C., Suh C. S., \& Kim, S. H. (2009). Impact of blastocyst transfer on offspring sex ratio and the monozygotic twinning rate: A systematic review and meta-analysis. Fertility and Sterility, 91, 2381-2390.
Chow, J. S., Benson, C. B., Racowsky, C., Doubilet, P. M., \& Ginsburg, E. (2001). Frequency of a monochorionic pair in multiple gestations: Relationship to mode of conception. Journal of Ultrasound in Medicine, 20, 757-760.

Delrieu, D., Himaya, E., Phillips, S., \& Kadoch, I. J. (2012). Monozygotic multiple pregnancies following IVF: A case report series of rare experience. Reproductive Biomedicine Online, 25, 460-465.

Fellman, J., \& Eriksson, A. W. (2006). Weinberg's differential rule reconsidered. Human Biology, 78, 253-275.

Franasiak, J. M., Dondik, Y., Molinaro, T. A., Hong, K. H., Forman, E. J., Werner, M. D., ... Scott, R. T., Jr. (2015). Blastocyst transfer is not associated with increased rates of monozygotic twins when controlling for embryo cohort quality. Fertility and Sterility, 103, 95-100.

Gee, R. E., Dickey, R. P., Xiong, X., Clark, L. S., \& Pridjian, G. (2014). Impact of monozygotic twinning on multiple births resulting from in vitro fertilization in the United States, 2006-2010. American Journal of Obstetrics and Gynecology, 210, e461-466.

Ghalili, A., McLennan, A., Pedersen, L., Kesby, G., \& Hyett, J. (2013). Outcomes of monochorionic diamniotic twin pregnancies: A comparison of assisted and spontaneous conceptions. Australian and New Zealand Journal of Obstetrics and Gynaecology, 53, 437-442.

Hall, J. G. (2003). Twinning. Lancet, 362, 735-743.

Hardin, J., Selvin, S., Carmichael, S. L., \& Shaw, G. M. (2009). The estimated probability of dizygotic twins: A comparison of two methods. Twin Research and Human Genetics, 12, 79-85.

Imaizumi, Y., \& Nonaka, K. (1997). The twinning rates by zygosity in Japan, 1975-1994. Acta Geneticae Medicae et Gemellologiae (Roma), 46, 9-22.

Kanter, J. R., Boulet, S. L., Kawwass, J. F., Jamieson, D. J., \& Kissin, D. M. (2015). Trends and correlates of monozygotic twinning after single embryo transfer. Obstetrics \& Gynecology, 125, 111-117.

Knopman, J., Krey, L. C., Lee, J., Fino, M. E., Novetsky, A. P., \& Noyes, N. (2010). Monozygotic twinning: An eight-year experience at a large IVF center. Fertility and Sterility, 94, 502-510.

Knopman, J. M., Krey, L. C., Oh, C., Lee, J., McCaffrey, C., \& Noyes, N. (2014). What makes them split? Identifying risk factors that lead to monozygotic twins after in vitro fertilization. Fertility and Sterility, 102, 82-89.

Luke, B., Brown, M. B., Wantman, E., \& Stern, J. E. (2014). Factors associated with monozygosity in assisted reproductive technology pregnancies and the risk of recurrence using linked cycles. Fertility and Sterility, 101, 683-689.

Nakasuji, T., Saito, H., Araki, R., Nakaza, A., Nakashima, A., Kuwahara, A., ... Sakumoto, T. (2014). The incidence of monozygotic twinning in assisted reproductive technology: analysis based on results from the 2010 Japanese ART national registry. Journal of Assisted Reproduction and Genetics, 31, 803-807.

Ortibus, E., Lopriore, E., Deprest, J., Vandenbussche, F. P., Walther, F. J., Diemert, A., ... Lewi, L. (2009). The pregnancy and long-term neurodevelopmental outcome of 
monochorionic diamniotic twin gestations: A multicenter prospective cohort study from the first trimester onward. American Journal of Obstetrics and Gynecology, 200, e491498.

Osianlis, T., Rombauts, L., Gabbe, M., Motteram, C., \& Vollenhoven, V. (2014). Incidence and zygosity of twin births following transfers using a single fresh or frozen embryo. Human Reproduction, 29, 1438-1443.

Parazzini, F., Cipriani, S., Bulfoni, G., Bulfoni, C., Frigerio, A., Somigliana, E., \& Mosca, F. (2015). The risk of birth defects after assisted reproduction. Journal of Assisted Reproduction and Genetics, 32, 379-385.

Ren, X., Liu, Q., Chen, W., Zhu, G., \& Zhang, H. (2013). Effect of the site of assisted hatching on vitrified-warmed blastocyst transfer cycles: A prospective randomized study. Journal of Assisted Reproduction and Genetics, 30, 691697.

Sobek, A., Jr., Zborilova, B., Prochazka, M., Silhanova, E., Koutna, O., Klaskova, E., ... Sobek, A. (2015). High incidence of monozygotic twinning after assisted reproduction is related to genetic information, but not to assisted reproduction technology itself. Fertility and Sterility, 103, 756-760.

Sperling, L., Kiil, C., Larsen, L. U., Qvist, I., Schwartz, M., Jorgensen, C., ... Tabor, A. (2006). Naturally conceived twins with monochorionic placentation have the highest risk of fetal loss. Ultrasound in Obstetrics and Gynecology, 28, 644-652.

Tocino, A., Blasco, V., Prados, N., Vargas, M. J., Requena, A., Pellicer, A., \& -Sanchez, M. (2015). Monozygotic twinning after assisted reproductive technologies: A case report of asymmetric development and incidence during 19 years in an international group of in vitro fertilization clinics. Fertility and Sterility, 103, 1185-1189.

Vitthala, S., Gelbaya, T. A., Brison, D. R., Fitzgerald, C. T., \& Nardo, L. G. (2009). The risk of monozygotic twins after assisted reproductive technology: A systematic review and meta-analysis. Human Reproduction Update, 15, 45-55.

Weinberg, W. (1901). Beitrage zur physiologie und pathologie der mehrlingsgeburten beim Menschen. Pflugers Archiv für Gesamte Pysiologie, 88, 346-350. 